\title{
Comparative Efficacy of Ciltacabtagene Autoleucel in CARTITUDE-1 vs Physician's Choice of Therapy in the Long-Term Follow-Up of POLLUX, CASTOR, and EQUULEUS Clinical Trials for the Treatment of Patients with Relapsed or Refractory Multiple Myeloma
}

\author{
Katja Weisel ${ }^{1} \cdot$ Thomas Martin $^{2} \cdot$ Amrita Krishnan $^{3} \cdot$ Sundar Jagannath $^{4} \cdot$ Anil Londhe $^{5} \cdot$ Sandhya Nair $^{6} \cdot$ Joris Diels $^{7}$. \\ Martin Vogel $^{8}$. Jordan M. Schecter ${ }^{9} \cdot$ Arnob Banerjee $^{10}$. Jesus G. Berdeja ${ }^{11} \cdot$ Tonia Nesheiwat $^{12} \cdot$ Ashraf Garrett $^{12}$. \\ Keqin $\mathrm{Qi}^{13}$. Satish Valluri ${ }^{14}$. Saad Z. Usmani ${ }^{15} \cdot$ Kwee Yong $^{16}$
}

Accepted: 2 November 2021 / Published online: 25 November 2021

(C) The Author(s) 2021

\begin{abstract}
Background and Objective Ciltacabtagene autoleucel (cilta-cel) is a novel agent being investigated in the single-arm CARTITUDE-1 trial (NCT03548207) for patients with relapsed or refractory multiple myeloma who are triple-class exposed to an immunomodulatory drug, proteasome inhibitor, and an anti-CD38 monoclonal antibody. The objective of this study was to evaluate the comparative efficacy of cilta-cel vs physician's choice of treatment, as no head-to-head trials have been conducted. Methods An external control arm for CARTITUDE-1 was created from patients in the long-term follow-up for three clinical trials of daratumumab (POLLUX, CASTOR, and EQUULEUS) who satisfied the eligibility criteria of CARTITUDE-1. These patients received physician's choice of treatment following the discontinuation of study drugs. Inverse probability of treatment weighting was used to align the external control and CARTITUDE-1 populations on important baseline characteristics. Overall response rate, complete response or better rate, progression-free survival, time to next treatment, and overall survival were assessed. Several sensitivity analyses were conducted.

Results After propensity score weighting, baseline characteristics were comparable between cohorts. Patients showed improved results with cilta-cel vs physician's choice of treatment: overall response rate (relative risk: 2.95 [95\% confidence interval (CI) 2.27, 3.84; $p<0.0001]$ ), complete response or better (relative risk: 111.70 [95\% CI 29.08, 429.06; $p<0.0001]$ ), progression-free survival (hazard ratio [HR]: 0.24 [95\% CI 0.15, 0.37; $p<0.0001]$ ), time to next treatment (HR: 0.14 [95\% CI 0.09, 0.22; $p<$ 0.0001]), and overall survival (HR: 0.21 [95\% CI 0.13, $0.35 ; p<0.0001]$ ). Results were consistent across all sensitivity analyses. Conclusions Cilta-cel showed superior efficacy compared with physician's choice of treatment, making it a promising new treatment option for patients with triple-class exposed relapsed or refractory multiple myeloma.
\end{abstract}

\section{Introduction}

Multiple myeloma (MM) is an incurable hematological malignancy with a rapidly evolving treatment landscape [1]. The past decade has seen the introduction of new agents in the treatment classes of immunomodulatory drugs (IMiDs) and proteasome inhibitors (PIs), as well as a novel treatment class, monoclonal antibodies (MoABs) [1]. Despite the improved outcomes provided by these treatments, most

Katja Weisel

k.weisel@uke.de

Extended author information available on the last page of the article

\section{Key Points}

Ciltacabtagene autoleucel (cilta-cel; CARTITUDE-1) and physician's choice of treatment (post-trial data for three daratumumab clinical trials, POLLUX, CASTOR, and EQUULEUS) were indirectly compared given the absence of direct head-to-head trials.

Cilta-cel demonstrated statistically and clinically superior efficacy results compared to physician's choice of treatment. Based on these findings, cilta-cel offers substantial clinical benefits for patients with triple-class exposed relapsed or refractory multiple myeloma. 
patients with MM relapse, undergoing cycles of worsening disease and reduced rate and duration of response with each successive line of therapy (LOT) [2]. For patients with relapsed or refractory multiple myeloma (RRMM) who have been exposed to IMiDs, PIs, and anti-CD38 MoABs (i.e., triple-class exposed), there is no clear standard of care and treatment burden is often high [3]. Median progression-free survival (PFS) and overall survival (OS) remain poor, with values of 3-6 months and less than 12 months, respectively $[4,5]$.

Ciltacabtagene autoleucel (cilta-cel; JNJ-68284528) is a chimeric antigen receptor T-cell (CAR-T) therapy that targets the B-cell maturation antigen (BCMA), a key marker of myeloma cells. Cilta-cel is currently being investigated in the open-label, single-arm CARTITUDE-1 trial (NCT03548207) for patients with RRMM who are tripleclass exposed [6]. Based on favorable results from CARTITUDE-1 [6, 7], cilta-cel was accepted for an accelerated assessment by the European Medicines Agency in February 2021 and the US Food and Drug Administration has granted priority review $[8,9]$. However, given the heterogeneity in currently used treatments and the observed poor outcomes in triple-class exposed patients, no randomized clinical trials have compared cilta-cel directly with currently used treatments.

Given the absence of a comparator arm in CARTITUDE-1, an external control arm may be used to establish comparative efficacy of cilta-cel vs currently used treatments. One appropriate source for such an external control is the long-term follow-up data from three clinical trials of the anti-CD38 MoAB daratumumab: POLLUX (NCT02076009) [10], CASTOR (NCT02136134) [11], and EQUULEUS (NCT01998971) [12], wherein patients received physician's choice of therapy after discontinuing the trial treatments. Indirect treatment comparison methods that adjust for differences in patient populations can be used to conduct a hypothetical head-to-head comparison [13]. The objective of this study was to evaluate the comparative efficacy of cilta-cel from CARTITUDE-1 vs physician's choice of treatment from the long-term follow-up of the POLLUX, CASTOR, and EQUULEUS global clinical trials for patients with triple-class exposed RRMM.

\section{Participants and Methods}

\subsection{Data Sources}

\subsubsection{CARTITUDE-1}

CARTITUDE-1 is a multi-center, phase Ib/II, open-label, single-arm study that recruited patients into its US cohort between July 2018 and October 2019. T cells from enrolled patients were obtained through leukapheresis and used to produce cilta-cel, which was administered via an infusion. Full details for CARTITUDE-1 have been previously published [6]. The present analysis was based on an updated data cut-off of February 2021, corresponding to a median follow-up of 18 months [7].

\subsubsection{Daratumumab Clinical Trials}

This study used long-term follow-up data from three daratumumab RRMM clinical trials: POLLUX, CASTOR, and EQUULEUS, which provided information for a large cohort of predominantly ex-US and triple-class exposed patients treated with physician's choice of therapy after discontinuing the trial treatments [3]. POLLUX and CASTOR were openlabel, randomized, phase III trials evaluating the efficacy of daratumumab in combination with lenalidomide-dexamethasone and bortezomib-dexamethasone, respectively $[14,15]$. EQUULEUS was an open-label, non-randomized, phase Ib trial evaluating the safety, tolerability, and dose regimen of daratumumab and various combination therapies [16]. Full study details for POLLUX, CASTOR, and EQUULEUS have been previously published [14-16]. Data cut-off for patients included in the present analysis was September 2019 for POLLUX and CASTOR and ranged from 2017 to 2019 for EQUULEUS (depending on study arm), corresponding to an overall median follow-up of 16.9 months across all eligible LOTs received since patients became triple-class exposed.

\subsection{Study Population and Design}

Individual patient-level data were available from all data sources. The main analysis included the treated population of CARTITUDE-1, which consisted of patients who were infused with cilta-cel (median of 47 days after apheresis). Patients from the daratumumab clinical trials were included in the external control arm (referred to as the physician's choice cohort) if they met the key inclusion criteria of CARTITUDE-1 (i.e., triple-class exposed, at least three prior LOTs, ${ }^{1}$ Eastern Cooperative Oncology Group score less than two, creatinine less than or equal to $2 \mathrm{mg} / \mathrm{dL},{ }^{2}$ and disease progression within 12 months of the most recent LOT). Patients in the physician's choice cohort also must have received the physician's choice treatment after triple class exposure. Patients in the physician's choice cohort

\footnotetext{
${ }^{1}$ CARTITUDE-1 inclusion criteria required three or more prior LOTs or double refractoriness to an IMiD and a PI; however, all enrolled patients received three or more prior LOTs.

${ }^{2}$ CARTITUDE-1 inclusion criterion was creatinine clearance of $\geq 40$ $\mathrm{mL} / \mathrm{min} / 1.73 \mathrm{~m}^{2}$; however, all enrolled patients had creatinine levels $\leq 2 \mathrm{mg} / \mathrm{dL}$.
} 
were reweighted to make their baseline characteristics similar to those of patients in CARTITUDE-1, using average treatment effect in the treated weights derived from propensity scores. This emulated a hypothetical comparative trial in which patients were randomized to receive cilta-cel (represented by CARTITUDE-1) or physician's choice of treatment (represented by the physician's choice cohort).

Because patients from the long-term follow-up data from the daratumumab clinical trials were retrospectively enrolled into this comparison, the first LOT initiated by a patient after they met the key eligibility criteria for CARTITUDE- 1 could be identified. This differed from CARTITUDE-1, in which cilta-cel is the treatment of interest and patients may have received additional LOTs between first meeting all eligibility criteria and entering the clinical trial. To account for this difference, if a patient in the physician's choice cohort received multiple LOTs after becoming eligible, that patient contributed multiple observations (corresponding to all eligible LOTs) to the present analysis, provided that the patient was eligible at the start of each LOT. Prior studies have shown this approach to be associated with improved statistical efficiency compared with restricting inclusion to only a single eligible LOT (e.g., first or last) [17, 18]. An exploratory analysis using only the first eligible LOT received by patients in the physician's choice cohort was conducted to assess potential differences in the two approaches.

To avoid survivorship bias in favor of cilta-cel, treatment lines were excluded from the physician's choice cohort if a patient experienced progressive disease or death within 47 days of initiating the corresponding LOT, thereby mimicking the median period of 47 days between apheresis and infusion in CARTITUDE-1 [17]. Index dates were defined for treated patients in CARTITUDE- 1 as the time of the cilta-cel infusion, and as 47 days after starting the relevant LOT for observations in the physician's choice cohort.

\subsection{Baseline Characteristics for Population Alignment}

Comparative efficacy estimates can be biased when baseline characteristics that are prognostic of the outcomes differ across non-randomized study cohorts and are left unadjusted [19]. In this study, a clinician-driven process was used to select the most important baseline characteristics for adjustment. First, a list of potential factors for adjustment was identified a priori based on studies from a literature review of the clinical evidence associated with triple-class exposed RRMM. This list was presented to a panel of five independent clinical hematology experts who lead clinical and research initiatives related to $\mathrm{MM}$ at major centers in the UK, Germany, and the USA, and modified according to their input. Next, clinicians were asked to rank the variables in order of importance for adjustment. To aid in the process, clinicians were provided with results from univariate regression analyses evaluating the prognostic strength of each factor in terms of PFS and OS in CARTITUDE-1. Clinicians were also provided with the standardized mean differences (SMDs) between each factor in CARTITUDE-1 and the physician's choice cohort (small, moderate, and substantial differences were represented by SMDs $\leq 0.1,>0.1$ and $\leq 0.2$, and $>0.2$, respectively [20]). Rankings were revised iteratively until a consensus was reached. The panel determined that refractory status, cytogenetic profile, International Staging System (ISS) stage, extramedullary plasmacytomas, time to progression on last regimen, number of prior LOTs, years since MM diagnosis, and age represented the minimum set of variables that should be adjusted for in all analyses to ensure clinical validity. Hence, these variables comprised the "base case". Remaining variables were ranked in order of importance (Table S1 in the Electronic Supplementary Material [ESM]).

\subsection{Outcomes}

Overall response rate (ORR), complete response (CR) or better rate ( $\geq$ CR rate), PFS, time to next treatment (TTNT), and OS were assessed. Response and progression were evaluated by an independent review committee in CARTITUDE-1 and by an investigator assessment in the physician's choice cohort. Overall response rate was calculated as the proportion of patients who achieved a partial response or better according to International Myeloma Working Group (IMWG) criteria [21]. The $\geq \mathrm{CR}$ rate was defined as the percentage of patients who achieved a CR or stringent CR according to IMWG criteria [21]. Progression-free survival was defined as the duration from the index date to the date of progression or death, whichever occurred first. For patients who had not progressed and were alive at the data cut-off, data were censored at the last disease evaluation before the start of any subsequent antimyeloma therapy or the retreatment of cilta-cel (CARTITUDE-1) and at the last follow-up date (physician's choice cohort). Time to next treatment was defined as the time from the index date to the initiation of the next LOT or death, whichever occurred first, with the last known date alive used in censoring. Overall survival was derived as the duration from the index date to the date of death. Patients who remained alive at the data cut-off or had an unknown vital status were censored at the last known date alive. Efficacy outcomes were evaluated monthly in CARTITUDE-1, every 12 weeks for post-trial treatments received in POLLUX and EQUULEUS (every 6 months as of May 2018), and every 16 weeks for post-trial treatments received in CASTOR. 


\subsection{Statistical Analysis}

Inverse probability of treatment weighting (IPTW) was conducted to adjust for baseline differences between patient cohorts [13]. Propensity scores were calculated using a logistic regression model that predicted assignment in the CARTITUDE- 1 cohort as a function of baseline covariates. Average treatment effect in the treated weighting was used, wherein patients from CARTITUDE-1 were kept as observed (i.e., assigned a weight of one), whereas patients in the physician's choice cohort were assigned a weight of $p /$ $(1-p)$, where $p$ is the propensity score predicting inclusion in the CARTITUDE-1 cohort [13]. Consequently, patients in the physician's choice cohort who had similar characteristics to CARTITUDE-1 were given a larger weight, making the physician's choice cohort similar to CARTITUDE-1. The effective sample size (ESS) was calculated to reflect the impact of weighting on the available information in the individual patient-level data [22]. The ESS is equivalent to the number of non-weighted observations needed to produce an effect estimate with the same precision as the indirect treatment comparison, had they been treated in a head-tohead trial [23].

For all outcomes, comparative efficacy was assessed for the unadjusted comparison (i.e., cilta-cel vs physician's choice of treatment prior to IPTW) and for the adjusted comparison (i.e., with IPTW). For response outcomes (ORR and $\geq \mathrm{CR}$ rate), odds ratios and $95 \%$ confidence intervals (CIs) were estimated using logistic regression (with weights applied for the adjusted comparison). The corresponding relative risk (RR) of response and 95\% CIs were derived from a Cox regression model with a constant in the time variable (with weights applied for the adjusted comparison) [24]. For time to event outcomes (PFS, TTNT, and OS), hazard ratios (HRs) and 95\% CIs were derived from a Cox proportional hazards model (with weights applied for the adjusted comparison). Irrespective of outcome, in the adjusted comparisons, the models also adjusted for the selected covariates, producing doubly robust results [25]. The cluster-robust sandwich variance estimator was used to account for within-person clustering of observations arising from the inclusion of all eligible LOTs in the physician's choice cohort. The proportional hazards assumption for time to event outcomes was confirmed by a visual inspection of the log-cumulative hazard plot.

For the physician's choice cohort, variables with missing values (applicable to ISS stage only) were imputed using the most commonly reported category. Imputation was not necessary for the CARTITUDE-1 data, as there were no missing values. Statistical analyses and graphical interpretation were conducted using R version 4.0.3 (R Foundation for Statistical Computing, Vienna, Austria).

\subsection{Sensitivity and Subgroup Analyses}

Four separate sensitivity analyses and one subgroup analysis were conducted to assess the impact of modifying the patient population, statistical methods, covariates, and outcome definitions. For each analysis, one of these criteria was modified, while all other specifications remained consistent with those outlined in the preceding sections. A comprehensive overview of each analysis is provided in Table S2 in the ESM.

For the first sensitivity analysis, the patient population was expanded to all 113 enrolled patients in CARTITUDE-1, including patients who underwent apheresis but withdrew prior to the cilta-cel infusion. To make the physician's choice cohort comparable to the enrolled population of CARTITUDE-1, all events from the initiation of physician's choice of treatment were included. In the second analysis, a multivariable regression model that included the base-case covariates was conducted without the use of weights. The third analysis adjusted for the following variables: hemoglobin level, prior stem cell transplant, Eastern Cooperative Oncology Group status, race, sex, and type of MM, in addition to the base-case variables. The fourth analysis used a modified definition of PFS in the physician's choice cohort, defined as the time from the index date to disease progression, switch to subsequent treatment, or death due to any cause, whichever occurred first. Last, a subgroup analysis was conducted considering only patients from the treated population of CARTITUDE-1 and the physician's choice cohort who were penta-refractory (to at least two IMiDs, two PIs, and an anti-CD38 MoAB).

\subsection{Research Ethics Statement}

The CARTITUDE-1 trial protocol was reviewed and approved by an independent ethics committee/institutional review board at all participating sites. All patients participating in the trial provided written informed consent. Similarly, the protocols of the clinical trials of daratumumab (POLLUX [10], CASTOR [11], and EQUULEUS [12]) were approved by local or independent institutional review boards or ethics committees at participating sites and all patients provided written informed consent. The current analyses were conducted in accordance with a protocol and statistical analysis plan developed prior to the start of data analysis.

\section{Results}

\subsection{Adjustment for Imbalances Between Cohorts}

The main analysis consisted of the treated population of CARTITUDE-1 $(N=97)$ and patients from the 
Fig. 1 Flow chart of patient selection. ${ }^{\mathrm{a} C A R T I T U D E}-1$ inclusion criteria required at least three prior line of therapies (LOTs) or double refractoriness to an immunomodulatory drug and a proteasome inhibitor; however, all enrolled patients received at least three prior LOTs. ${ }^{\mathrm{b}}$ CARTITUDE- 1 inclusion criterion was creatinine clearance of $\geq 40 \mathrm{~mL} / \mathrm{min} / 1.73$ $\mathrm{m}^{2}$; however, all enrolled patients had creatinine levels $\leq$ $2 \mathrm{mg} / \mathrm{dL}$. ECOG Eastern Cooperative Oncology Group, $N_{O B S}$ number of observations
CARTITUDE-1 COHORT

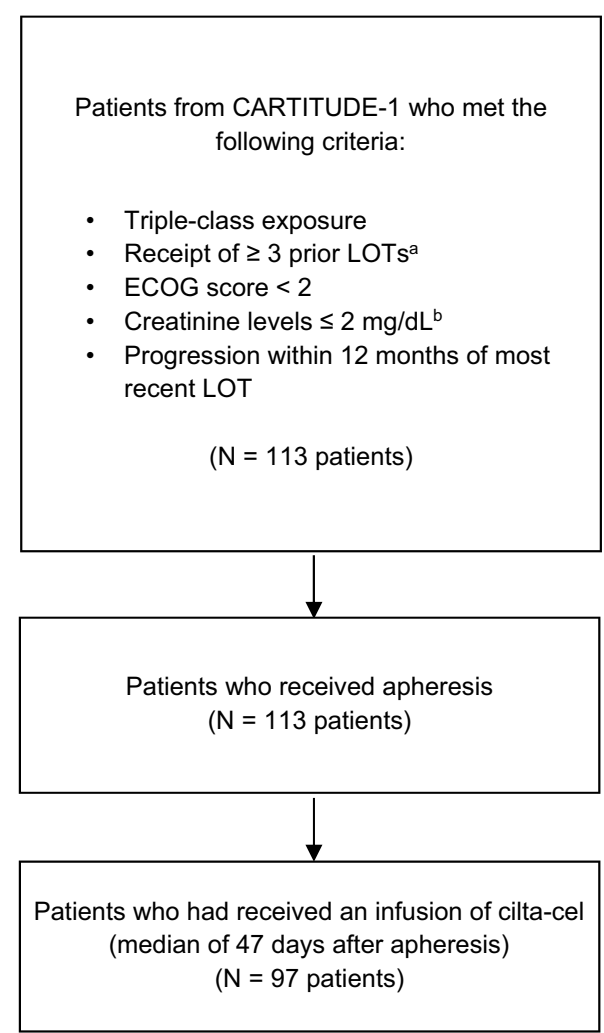

PHYSICIAN'S CHOICE COHORT

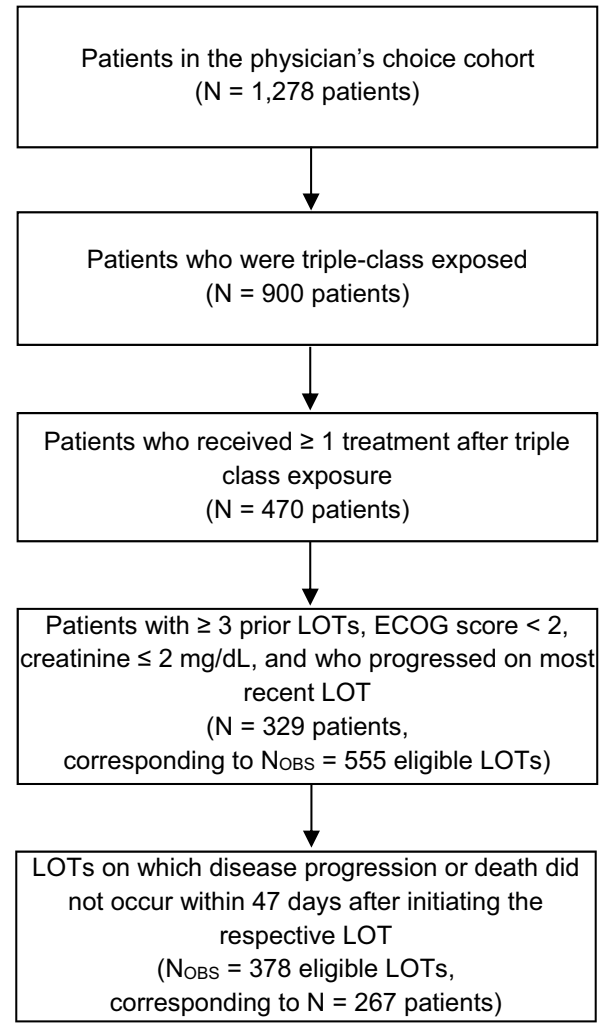

daratumumab clinical trials who were eligible for inclusion in the physician's choice cohort $(N=267)$. Patients in the physician's choice cohort contributed a total of 378 unique observations, corresponding to all eligible LOTs (Fig. 1). After adjustment with IPTW, the ESS of all observations included in the physician's choice cohort was 110 .

Baseline characteristics before and after adjustment with IPTW are shown in Table 1. Before adjustment, the CARTITUDE-1 population had a higher proportion of patients with ISS stage I, high-risk cytogenetics (at least one of del17p, $\mathrm{t}[4 ; 14]$, or $\mathrm{t}[14 ; 16])$, progression within 4 months on their last treatment regimen, extramedullary plasmacytomas, more than four prior LOTs, and a higher proportion of patients who were penta-refractory (to at least two IMiDs, two PIs, and an anti-CD38 MoAB) and younger than 65 years of age. After adjustment, the overall balance in baseline characteristics between populations was improved, with the mean SMD reduced from 0.33 to 0.16 . A plot showing the SMDs before and after adjustment is provided in Fig. $\mathrm{S} 1$ in the ESM.

Patients in the physician's choice cohort received 151 unique treatment combinations after becoming triple-class exposed. Treatments received alone or in combination included IMiDs (pomalidomide, thalidomide, and lenalidomide), PIs (carfilzomib, bortezomib, and ixazomib), and MoABs (daratumumab, elotuzumab, and isatuximab).
A full list of treatments is provided in Table 2. Patients in the physician's choice cohort were predominantly from the European Union (70.0\%), although patients were also included from North America (14.2\%), Asia/Pacific (12.7\%), and other regions $(3.0 \%)$.

\subsection{Comparative Efficacy Results}

Comparative efficacy results for response (ORR and $\geq \mathrm{CR}$ rate) and survival (PFS, TTNT, and OS) outcomes before and after adjustment are shown in Tables 3 and 4, respectively. Results were statistically significant in favor of ciltacel after adjustment, with cilta-cel substantially out-performing physician's choice of treatment. Patients treated with cilta-cel were 2.95-fold more likely to achieve an overall response and 111.70 -fold more likely to achieve $\geq \mathrm{CR}$ than patients treated with physician's choice of treatment (with $\geq \mathrm{CR}$ rates of $80.4 \%$ observed in CARTITUDE- 1 and $0.7 \%$ after adjustment in the physician's choice cohort). Cilta-cel reduced the risk of disease progression or death by approximately $76 \%$, the risk of initiating a subsequent treatment by approximately $86 \%$, and the risk of death by approximately $79 \%$.

Kaplan-Meier plots for PFS, TTNT, and OS are provided in Fig. 2. Patients treated with cilta-cel had a median PFS of 22.8 months (95\% CI 22.8, not reached); however, this 
Table 1 Overview of baseline characteristics before and after adjustment with IPTW for the base case

\begin{tabular}{|c|c|c|c|c|}
\hline \multirow[t]{3}{*}{ Characteristic } & \multirow[t]{3}{*}{ Categories } & \multicolumn{2}{|l|}{ Unadjusted } & \multirow{3}{*}{$\begin{array}{l}\text { Adjusted } \\
\text { Physician's } \\
\text { choice cohort } \\
\text { ESS }^{\mathrm{a}} \%\end{array}$} \\
\hline & & \multirow{2}{*}{$\begin{array}{l}\text { CARTITUDE-1 } \\
N(\%)\end{array}$} & \multirow{2}{*}{$\begin{array}{l}\text { Physician's choice } \\
\text { cohort } \\
N_{\mathrm{OBS}}(\%)\end{array}$} & \\
\hline & & & & \\
\hline Total & - & 97 (100) & $378(100)$ & $110(100)$ \\
\hline \multirow[t]{3}{*}{ Refractory status ${ }^{\mathrm{b}} *$} & Penta $^{c}$ & $41(42.3)$ & 69 (18.3) & $47(42.8)$ \\
\hline & Triple or quad ${ }^{\mathrm{d}}$ & $44(45.4)$ & $292(77.2)$ & $50(45.9)$ \\
\hline & Others & $12(12.4)$ & $17(4.5)$ & $12(11.3)$ \\
\hline \multirow[t]{3}{*}{ ISS stage* } & I & $61(62.9)$ & $208(55.0)^{\mathrm{e}}$ & $68(61.9)$ \\
\hline & II & $22(22.7)$ & $128(33.9)^{\mathrm{e}}$ & $26(23.6)$ \\
\hline & III & $14(14.4)$ & $42(11.1)^{\mathrm{e}}$ & $16(14.4)$ \\
\hline \multirow[t]{3}{*}{ Cytogenetic profile* } & High risk ${ }^{\mathrm{f}}$ & $23(23.7)$ & $55(14.6)$ & $22(19.7)$ \\
\hline & Standard risk & $68(70.1)$ & $219(57.9)$ & $81(73.7)$ \\
\hline & Unknown & $6(6.2)$ & $104(27.5)$ & $7(6.5)$ \\
\hline \multirow{2}{*}{ Time to progression on last regimen* } & $\leq 4$ months & 48 (49.5) & $128(33.9)$ & $62(56.4)$ \\
\hline & $>4$ months & $49(50.5)$ & $250(66.1)$ & $48(43.6)$ \\
\hline \multirow[t]{2}{*}{ Extramedullary plasmacytoma ${ }^{\mathrm{g} *}$} & No & $84(86.6)$ & $358(94.7)$ & $89(80.9)$ \\
\hline & Yes & $13(13.4)$ & $20(5.3)$ & $21(19.1)$ \\
\hline \multirow[t]{2}{*}{ Number of prior LOTs* } & $\leq 4$ & $33(34.0)$ & $220(58.2)$ & $38(34.6)$ \\
\hline & $>4$ & $64(66.0)$ & $158(41.8)$ & $72(65.4)$ \\
\hline \multirow[t]{2}{*}{ Years since MM diagnosis* } & $<6$ & $45(46.4)$ & $160(42.3)$ & $56(50.9)$ \\
\hline & $\geq 6$ & $52(53.6)$ & $218(57.7)$ & $54(49.1)$ \\
\hline \multirow[t]{2}{*}{ Age* } & $<65$ & $62(63.9)$ & $153(40.5)$ & $69(62.3)$ \\
\hline & $\geq 65$ & $35(36.1)$ & $225(59.5)$ & $41(37.7)$ \\
\hline \multirow[t]{2}{*}{ Hemoglobin (g/dL) } & $<12$ & $90(92.8)$ & $212(56.1)$ & $52(47.2)$ \\
\hline & $\geq 12$ & $7(7.2)$ & $166(43.9)$ & $58(52.8)$ \\
\hline \multirow[t]{2}{*}{ LDH levels (units/L) } & $<280$ & $85(87.6)$ & NR & NR \\
\hline & $\geq 280$ & $12(12.4)$ & NR & NR \\
\hline \multirow[t]{2}{*}{ Prior stem cell transplant } & No & $10(10.3)$ & $88(23.3)$ & $18(16.2)$ \\
\hline & Yes & 87 (89.7) & $290(76.7)$ & $92(83.8)$ \\
\hline \multirow[t]{2}{*}{ ECOG status } & 0 & $39(40.2)$ & $166(43.9)$ & $52(47.7)$ \\
\hline & 1 & $58(59.8)$ & $212(56.1)$ & $58(52.3)$ \\
\hline \multirow[t]{3}{*}{ Race } & White & $69(71.1)$ & $299(79.1)$ & $92(83.7)$ \\
\hline & Black/African American & $17(17.5)$ & $19(5.0)$ & $6(5.2)$ \\
\hline & Not reported/other & $11(11.3)$ & $60(15.9)$ & $12(11.1)$ \\
\hline \multirow[t]{2}{*}{ Sex } & Female & $40(41.2)$ & $179(47.4)$ & $49(44.9)$ \\
\hline & Male & $57(58.8)$ & $199(52.6)$ & $61(55.1)$ \\
\hline \multirow[t]{3}{*}{ Type of MM } & $\mathrm{IgG}$ & $57(58.8)$ & $200(52.9)$ & $56(50.9)$ \\
\hline & Light chain & $24(24.7)$ & $80(21.2)$ & $29(26.5)$ \\
\hline & Other & $16(16.5)$ & 98 (25.9) & $25(22.7)$ \\
\hline
\end{tabular}

ECOG Eastern Cooperative Oncology Group, ESS effective sample size, IMiD immunomodulatory drug, IPTW inverse probability of treatment weighting, ISS International Staging System, $L D H$ lactate dehydrogenase, LOTs lines of therapy, $M M$ multiple myeloma, $M o A B$ monoclonal antibody, $N_{O B S}$ number of observations, $N R$ not reported, $P I$ proteasome inhibitor

*Variables used in adjustment with IPTW for the base case

${ }^{a}$ ESS was rounded to whole numbers

${ }^{\mathrm{b}}$ Refractoriness was defined as from the case report form as progressive disease/relapsed per investigator assessment (physician's choice cohort) and by International Myeloma Working Group consensus criteria (CARTITUDE-1) [6]

${ }^{\mathrm{c}}$ Refractory to at least two IMiDs, two PIs, and an anti-CD38 MoAB

${ }^{\mathrm{d}}$ Refractory to two IMiDs and one PI; or two PIs and one IMiD; or two IMiDs and two PIs

${ }^{\mathrm{e}}$ ISS imputed for 56 observations in the physician's choice cohort

${ }^{\mathrm{f}}$ At least one of del17p, $\mathrm{t}(14 ; 16)$, or $\mathrm{t}(4 ; 14)$

${ }^{\mathrm{g}}$ Refers to soft-tissue mass that is not in contact with bone; does not include bone-based plasmacytomas[35] 
Table 2 Physician's choice of treatment received across all eligible lines of therapy in the physician's choice cohort

\begin{tabular}{|c|c|c|c|c|}
\hline \multirow[t]{2}{*}{ Treatment } & \multicolumn{2}{|c|}{ Hierarchy $^{\mathrm{a}}$} & \multicolumn{2}{|c|}{$\begin{array}{l}\text { Received in any } \\
\text { eligible line of } \\
\text { therapy }^{\mathrm{b}}\end{array}$} \\
\hline & $N=378$ & $\%$ & $N=378$ & $\%$ \\
\hline Carfilzomib & 66 & 17.5 & 66 & 17.5 \\
\hline Pomalidomide & 89 & 23.5 & 98 & 25.9 \\
\hline Daratumumab & 13 & 3.4 & 29 & 7.7 \\
\hline Ixazomib & 17 & 4.5 & 21 & 5.6 \\
\hline Elotuzumab & 4 & 1.1 & 4 & 1.1 \\
\hline Bortezomib & 63 & 16.7 & 67 & 17.7 \\
\hline Lenalidomide & 36 & 9.5 & 68 & 18.0 \\
\hline Panobinostat & 0 & 0.0 & 15 & 4.0 \\
\hline Selinexor & 3 & 0.8 & 6 & 1.6 \\
\hline Isatuximab & 2 & 0.5 & 2 & 0.5 \\
\hline $\begin{array}{l}\text { Thalidomide, or melphalan, } \\
\text { or cyclophosphamide }^{c}\end{array}$ & 51 & 13.5 & 128 & 33.9 \\
\hline Dexamethasone & 6 & 1.6 & 289 & 76.5 \\
\hline Others ${ }^{\mathrm{d}}$ & 28 & 7.4 & 99 & 26.2 \\
\hline
\end{tabular}

${ }^{a}$ For each treatment, the number and percent represent the patients who received that treatment as a single-agent therapy or in combination with any of the other treatments listed in the subsequent rows

${ }^{b}$ Received alone or in combination; therefore, the total adds to more than $100 \%$ as treatments from the same line of therapy can be counted more than once

${ }^{c}$ Any one received alone or in combination with either one of the three or other drugs

d،"Others" were received in combination and included bendamustine hydrochloride, peginterferon alfa- $2 \mathrm{a}$, corticosteroids, cisplatin, cytarabine, etoposide, cobimetinib, venetoclax, doxorubicin, and investigational agents

was reached when few patients remained at risk and was expected to be underestimated. Still, this duration is more than four times as long as the median PFS in the adjusted physician's choice cohort (5.6 months). Neither the median TTNT nor OS was reached for patients treated in CARTITUDE-1. In contrast, the adjusted physician's choice cohort displayed a median TTNT and OS of 4.8 months and 10.9 months, respectively (Table 4).

The exploratory analysis that included only patients' first eligible LOT received in the physician's choice cohort demonstrated consistent results with those from the main analysis (Table 5; Fig. S2 in the ESM).

\subsection{Sensitivity and Subgroup Analyses}

In the sensitivity analysis that included all enrolled patients of CARTITUDE-1, findings were consistent with the main analysis, with statistically significant treatment effects in favor of cilta-cel observed for ORR (RR: 3.17 [95\% CI 2.48, 4.06; $p<0.0001]$ ), $\geq \mathrm{CR}$ rate (RR: 76.05 [95\% CI $31.42,184.07 ; p<0.0001]$ ), PFS (HR: 0.27 [95\% CI 0.18, 0.39; $p<0.0001]$ ), TTNT (HR: 0.16 [95\% CI 0.11, 0.23; $p<0.0001$ ) , and OS (HR: 0.26 [95\% CI 0.17, 0.40; $p<$ $0.0001]$ ) (Table 5). Findings from all other sensitivity analyses were also aligned with the main analysis, consistently showing a statistically significant benefit for cilta-cel over physician's choice of treatment. Similarly, in the subgroup analysis that considered penta-refractory patients, results were consistent with those from the main analysis (Tables S3-S4 and Fig. S3 in the ESM); however, the adjusted physician's choice subgroup had a low ESS of 28, limiting the validity of the adjusted indirect comparisons.

\section{Discussion}

As patients with RRMM advance through IMiDs, PIs, and anti-CD38 MoABs, it becomes difficult to select an appropriate next treatment regimen. There is no clear standard

Table 3 Rates and comparative efficacy of response outcomes for cilta-cel vs physician's choice of treatment

\begin{tabular}{|c|c|c|c|c|c|c|c|}
\hline & \multirow{2}{*}{$\begin{array}{l}\text { CARTITUDE-1 } \\
\text { Observed response } \\
(\%)\end{array}$} & \multicolumn{2}{|c|}{ Physician's choice cohort } & \multicolumn{2}{|c|}{ Unadjusted comparison } & \multicolumn{2}{|l|}{ Adjusted comparison $^{\mathrm{a}}$} \\
\hline & & $\begin{array}{l}\text { Observed } \\
\text { response } \\
(\%)\end{array}$ & $\begin{array}{l}\text { Adjusted }^{\mathrm{a}} \\
\text { response } \\
(\%)\end{array}$ & $\begin{array}{l}\mathrm{OR}^{\mathrm{b}}(95 \% \mathrm{CI}) \\
p \text {-value }\end{array}$ & $\begin{array}{l}\mathrm{RR}^{\mathrm{b}}(95 \% \mathrm{CI}) \\
p \text {-value }\end{array}$ & $\begin{array}{l}\mathrm{OR}^{\mathrm{b}}(95 \% \mathrm{CI}) \\
p \text {-value }\end{array}$ & $\begin{array}{l}\mathrm{RR}^{\mathrm{b}}(95 \% \mathrm{CI}) \\
p \text {-value }\end{array}$ \\
\hline ORR & 97.9 & 37.8 & 33.6 & $\begin{array}{c}78.06(24.20, \\
478.16),<0.0001\end{array}$ & $\begin{array}{l}2.59(2.26,2.96) \\
<0.0001\end{array}$ & $\begin{array}{l}133.01(35.71, \\
887.29),<0.0001\end{array}$ & $\begin{aligned} & 2.95(2.27,3.84), \\
< & 0.0001\end{aligned}$ \\
\hline$\geq \mathrm{CR}$ rate & 80.4 & 1.6 & 0.7 & $\begin{array}{l}254.53(105.87 \\
724.78),<0.0001\end{array}$ & $\begin{array}{l}50.66(22.69, \\
113.09),<0.0001\end{array}$ & $\begin{array}{l}754.27(114.19, \\
35258.00),<0.0001\end{array}$ & $\begin{array}{l}111.70(29.08, \\
429.06),<0.0001\end{array}$ \\
\hline
\end{tabular}

$C I$ confidence interval, $\geq C R$ complete response or better, $O R$ odds ratio, $O R R$ overall response rate, $R R$ relative risk

${ }^{a}$ Adjusted for refractory status, International Staging System stage, cytogenetic profile, time to progression on last regimen, extramedullary plasmacytoma, number of prior lines of therapy, years since multiple myeloma diagnosis, and age

${ }^{\mathrm{b}} \mathrm{ORs}$ and RRs $>1$ indicate a favorable treatment effect for cilta-cel 
Table 4 Estimated medians and comparative efficacy of survival outcomes for cilta-cel vs physician's choice of treatment

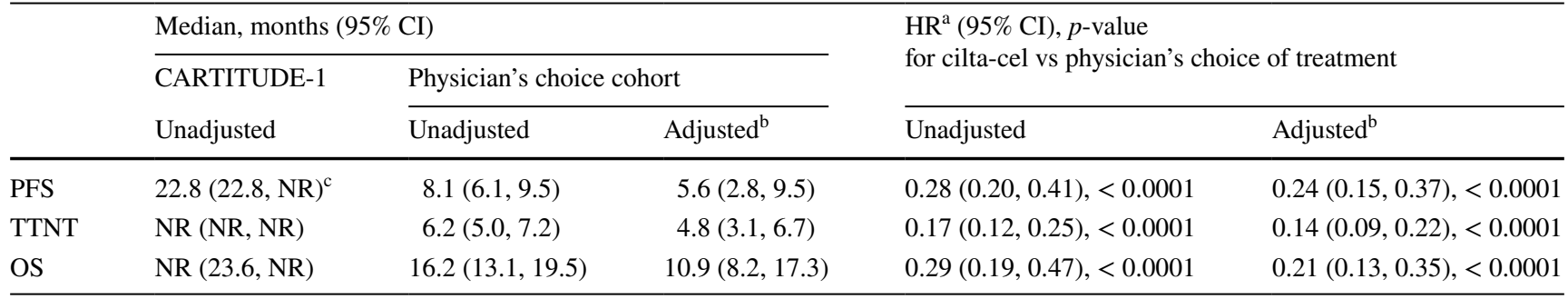

$C I$ confidence interval, $H R$ hazard ratio, $N R$ not reached, $O S$ overall survival, $P F S$ progression-free survival, TTNT time to next treatment ${ }^{a} \mathrm{HR}<1$ indicates a favorable treatment effect for cilta-cel

${ }^{\mathrm{b}}$ Adjusted for refractory status, International Staging System stage, cytogenetic profile, time to progression on last regimen, extramedullary plasmacytoma, number of prior lines of therapy, years since multiple myeloma diagnosis, and age

${ }^{\mathrm{c}}$ Median should be interpreted with caution, as reached when few patients were still at risk and may be an underestimate

A

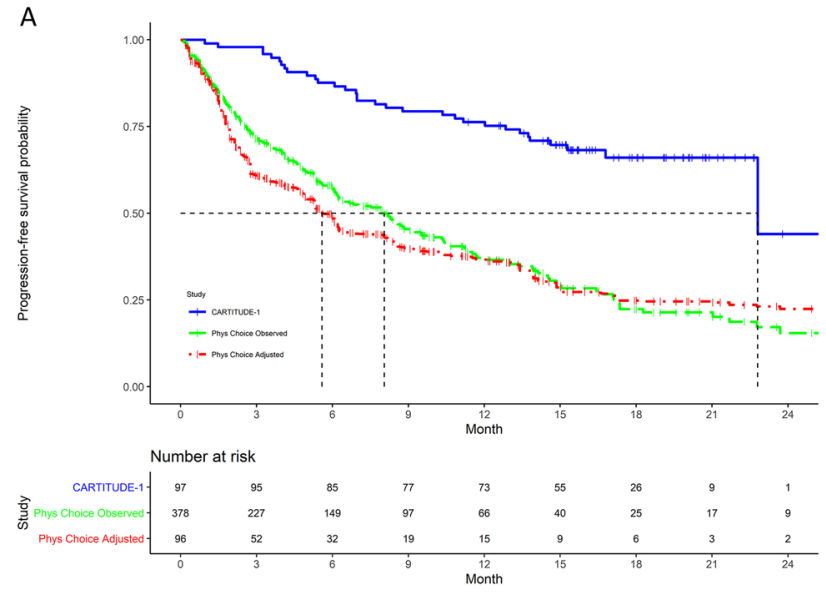

B

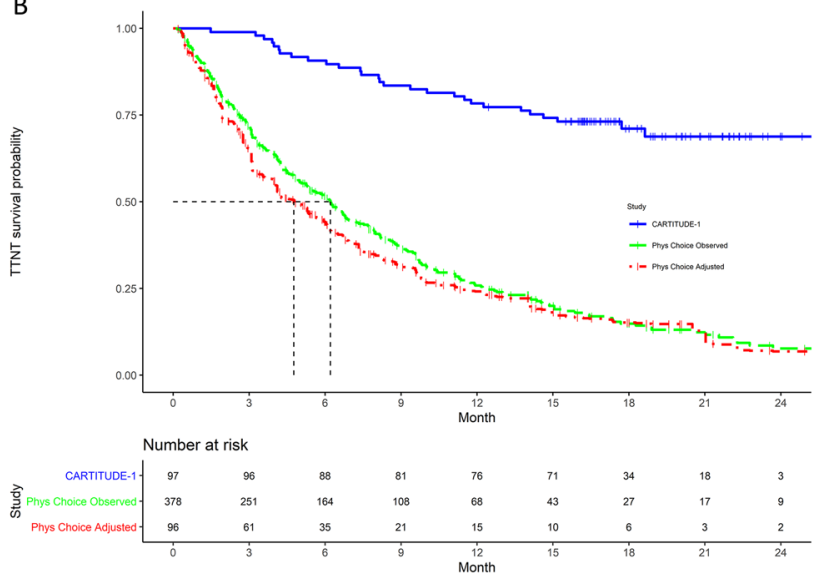

C
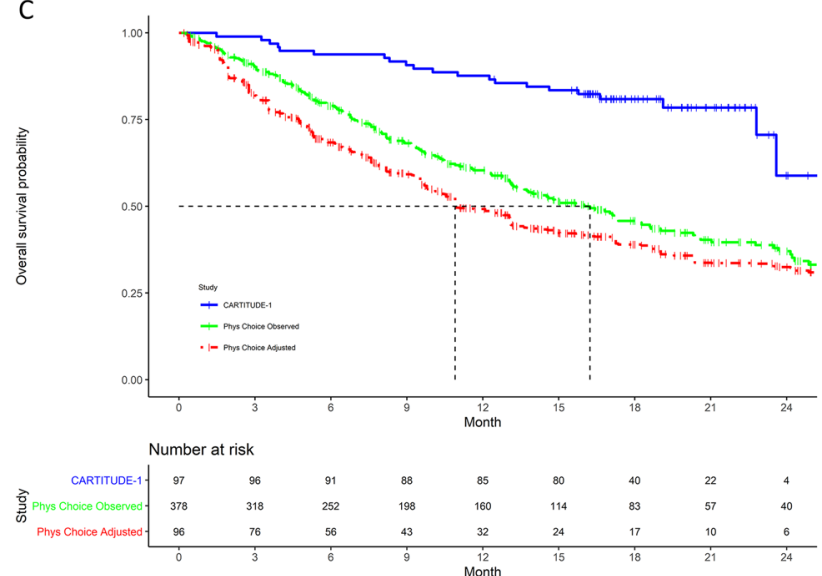

Fig. 2 Kaplan-Meier estimated A progression-free survival, B time to next treatment, and $\mathbf{C}$ overall survival for CARTITUDE-1 (observed) and the physician's choice cohort (observed and adjusted). Note: Number at risk for the adjusted physician's choice cohort represents the sum of the propensity score weights, not the effective sample size. Adjusted results correspond to the base-case scenario that adjusted for refractory status, cytogenetic profile, International Staging System stage, extramedullary plasmacytoma, time to progression on last regimen, number of prior lines of therapy, years since multiple myeloma diagnosis, and age. The adjusted curves reflect inverse probability of treatment weighting with average treatment effect in the treated weights (not doubly robust). Phys physician's 
Table 5 Comparative efficacy for ORR, $\geq$ CR rate, PFS, TTNT, and OS across additional analyses

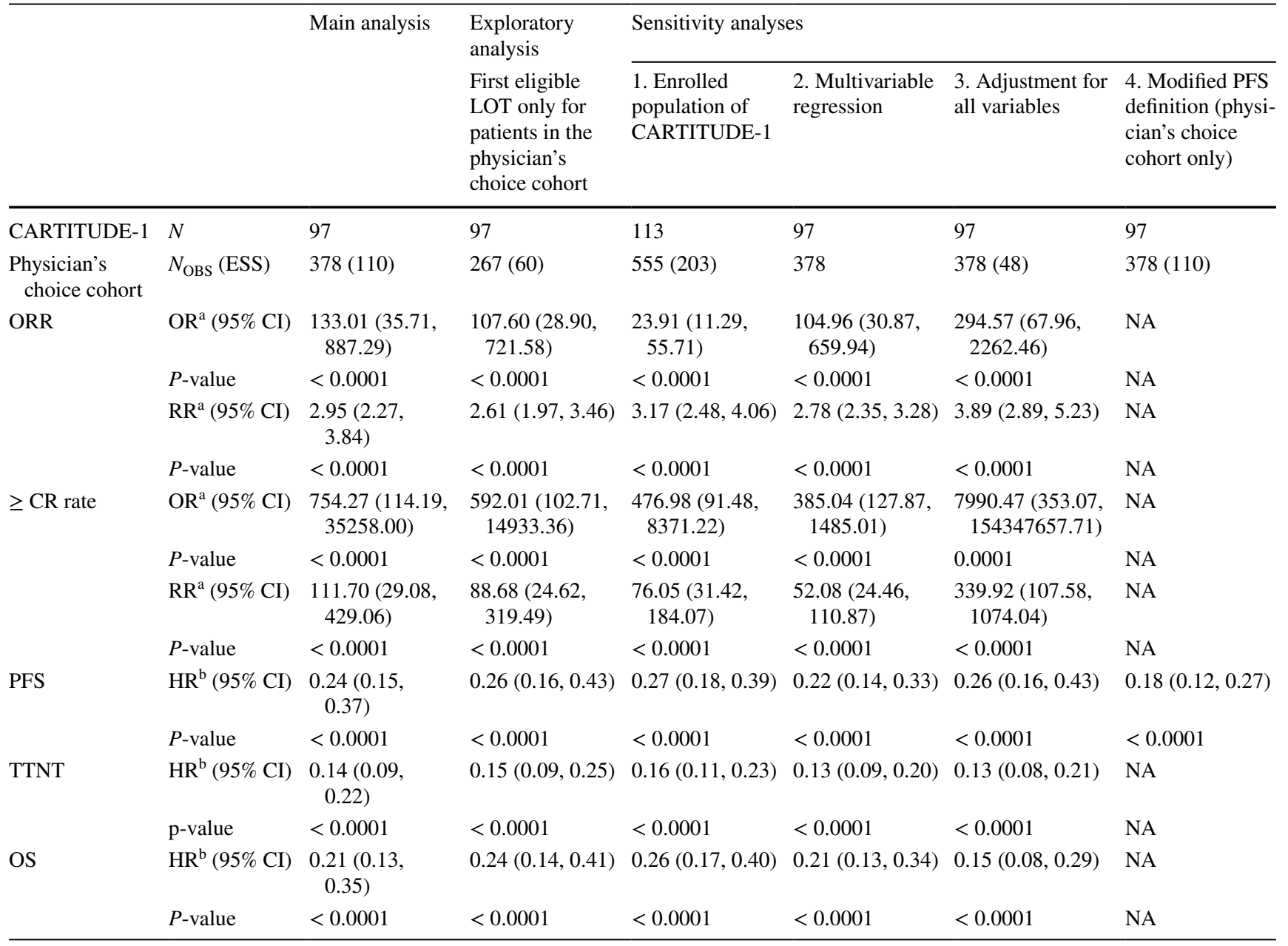

$C I$ confidence interval, $\geq C R$ complete response or better, ESS effective sample size, HR hazard ratio, LOT line of therapy, NA not applicable, $N_{O B S}$ number of observations, $O R$ odds ratio, $O R R$ overall response rate, $O S$ overall survival, $P F S$ progression-free survival, $R R$ relative risk, TTNT time to next treatment

The main analysis included the following specifications: treated population of CARTITUDE-1, inverse probability of treatment weighting, all eligible LOTs for patients in the physician's choice cohort, and adjustment for base-case variables (refractory status, cytogenetic profile, International Staging System stage, time to progression on last regimen, extramedullary plasmacytoma, number of prior lines of therapy, years since multiple myeloma diagnosis, and age). For each additional analysis, one of these specifications was modified, as outlined in Table S2 in the ESM ${ }^{\mathrm{a}} \mathrm{ORs}$ and RRs $>1$ indicate a favorable treatment effect for cilta-cel

${ }^{\mathrm{b}} \mathrm{HR}<1$ indicates a favorable treatment effect for cilta-cel

of care for patients with triple-class exposed RRMM, with at least 378 different treatment regimens comprising 48 different compounds currently being used to treat patients with this indication [3]. Given the vastness of the treatment landscape, emerging treatments such as cilta-cel, which has shown promise but has not been directly compared with currently available treatments, can further complicate the decisions faced by patients, clinicians, and payers. To address this knowledge gap, the present study conducted indirect treatment comparisons to estimate the comparative efficacy of cilta-cel vs physician's choice of treatment.
Indirect treatment comparison methods have been previously used to compare treatments for hematological malignancies, with recent publications related to RRMM, acute myeloid leukemia, and B-cell lymphoma [26-28]. The current analysis contributed to this growing body of evidence, but this time assessing the comparative efficacy of a novel BCMA-targeted CAR-T therapy in the setting of triple-class exposed RRMM. Cilta-cel demonstrated a statistically significant improvement over physician's choice of treatment for all outcomes of interest, with a large magnitude of effect observed in each instance. Additionally, main findings were consistent with those from sensitivity analyses conducted to 
assess the effect of varying the population, statistical methods, covariates, and outcome definitions used. Last, although the main analysis was unable to account for the sequence in which treatments were received by patients in the physician's choice cohort, consistent results were obtained in the exploratory analysis that considered only the first eligible LOT received by patients in the physician's choice cohort, providing confidence in the main findings.

The beneficial treatment effect demonstrated by cilta-cel in both CARTITUDE- 1 and the present comparative analysis is an important finding, and compares favorably with recent reports of the outcomes of newer agents in patients with triple-class exposed disease. Two such agents are belantamab mafodotin, an anti-BCMA antibody-drug conjugate, and selinexor, a selective inhibitor of nuclear export, which were assessed in DREAMM-2 and STORM, respectively [29-31]. In both studies, less than $35 \%$ of patients achieved an overall response and median PFS did not exceed 5 months [29-31]. A third novel agent, idecabtagene vicleucel, an anti-BCMA CAR-T therapy, has shown more promise, with an ORR of $73 \%$ and median PFS of 8.8 months [32]. In contrast, ciltacel demonstrated an ORR of $97.9 \%$ and a median PFS of 22.8 months (which may be underestimated as it was reached when few patients remained at risk) in CARTITUDE-1 [7].

The choice of external comparator for CARTITUDE-1 offered several strengths. The use of long-term follow-up data from several clinical trials provided a breadth of information on baseline patient characteristics and efficacy outcomes, including both response and disease progression, which are not available in some real-world datasets [33]. Moreover, the external comparator was restricted to patients from the daratumumab long-term follow-up clinical trials who satisfied key eligibility criteria of CARTITUDE-1, ensuring similarities between the cohorts. However, not all eligibility criteria for CARTITUDE-1 (i.e., blood count parameters and the level of measurable disease) were available in the daratumumab long-term clinical trials, which is acknowledged as a limitation of this analysis. Nonetheless, all key criteria that were available were applied, producing the most similar cohorts possible. Next, the daratumumab clinical trials represented a predominantly European population, whereas most available data on currently used treatments for patients with triple-class exposed RRMM are in the US setting $[4,5]$. Last, the response rates observed in the physician's choice cohort (ORR: $37.8 \%$; $\geq$ CR rate $1.6 \%$ ) were consistent with those reported in the prospective LocoMMotion study (ORR: $20.1 \%$; $\geq$ CR rate: $0.5 \%$ ) and the retrospective MAMMOTH study (ORR: $31.3 \%$; $\geq$ CR rate: $2.0 \%)[4,34]$, two key real-world studies in patients with triple-class exposed RRMM.

As in any non-randomized trial, the potential for residual confounding must be acknowledged. However, an evidenceinformed process was used to select the most important clinical factors that should be aligned between the two populations. Of the 15 factors identified a priori as important for population alignment, all factors aside from lactate dehydrogenase were available from both populations. Notably, lactate dehydrogenase was not among the eight top ranked factors, and therefore its unavailability did not impact the base-case analysis. Another potential limitation was that the method of PFS assessment differed between CARTITUDE-1 (independent review committee) and the physician's choice cohort (physician assessment). Consequently, the frequency of testing differed, and it is possible that progression events identified in the physician's choice cohort may not have satisfied IMWG criteria. However, the median PFS in the physician's choice cohort (8.1 months) was slightly longer than that reported for a similar patient population in the retrospective real-world MAMMOTH study (3.4 months), which used IMWG criteria [4]. Furthermore, a sensitivity analysis was conducted using a modified definition of PFS, which considered the earliest of disease progression or start of next treatment as an event. The start of a new treatment may have been more reliably recorded by the physician than progression, and patients at this late stage in their treatment journey are unlikely to initiate a new LOT for reasons other than disease progression. Findings from the sensitivity analysis were aligned with the main results, although the HR was slightly more in favor of cilta-cel.

Future analyses using real-world evidence can be used to validate the findings from the current study. Furthermore, whereas the unavailability of safety information for subsequent treatments received by patients in POLLUX, CASTOR, and EQUULEUS precluded an investigation of safety in the present study, real-world evidence can provide insight into the safety profile of cilta-cel vs physician's choice of treatment. Last, even though cilta-cel demonstrated superior efficacy to physician's choice of conventional treatments in this study period, similar to other CAR-Ts, cilta-cel can only be delivered in certified specialized treatment centers. Hence, real-world studies are needed to better understand referral patterns related to CAR-T therapies.

\section{Conclusions}

Comparative efficacy of cilta-cel vs physician's choice of conventional treatment received by a similar cohort of patients from three global clinical trials was assessed. Cilta-cel displayed a clinically and statistically significant improvement for all outcomes (ORR, $\geq \mathrm{CR}$ rate, PFS, TTNT, and OS), with similar results obtained across numerous sensitivity analyses. These findings suggest that cilta-cel may represent an important new treatment option for patients with triple-class exposed RRMM. 
Supplementary Information The online version contains supplementary material available at https://doi.org/10.1007/s40261-021-01100-y.

Acknowledgments Medical writing support was provided by EVERSANA. The authors acknowledge the contribution of Eloquent Scientific Solutions for the editorial support. The authors thank MarieKristin Leisten for her review and comments. She was employed by Janssen during the conduct of the study. The authors thank Imtiaz A. Samjoo, Anja Haltner, Krista Tantakoun, and Emily Rosta for revising the manuscript critically. All were employed by EVERSANA, Canada.

\section{Declarations}

Funding This work was supported by Janssen Pharmaceuticals and Legend Biotech.

Conflicts of interest/Competing interests $\mathrm{KW}$ received honoraria from and served in a consulting or advisory role for Adaptive Biotechonlogies, Amgen, BMS, Celgene, GSK, Janssen, Karyopharm Therapeutics, Oncopeptides, Roche/Genentech, Sanofi, and Takeda, served in a consulting or advisory role for GSK, received travel funding from Amgen, BMS, Celgene, GSK, Janssen, and Takeda, and received research funding from Amgen, Celgene, Janssen, and Sanofi. TM served in a consulting or advisory role for GlaxoSmithKline and Juno Therapeutics, and received research funding from Amgen, Janssen, and Sanofi. AK served in a consulting or advisory role for Adaptive Biotechnologies, Celgene/Bristol Meyers-Squibb, GlaxoSmithKline, Janssen Oncology, Pfizer, and Regeneron, served on speakers bureaus for Amgen, Celgene/Bristol Meyers-Squibb, GlaxoSmithKline and Takeda, served on scientific advisory boards for Sutro Biopharma, has equity in Celgene/Bristol Meyers-Squibb, and received research funding from Janssen Oncology. SJ is a consultant for Bristol Myers Squibb, Janssen, Karyopharm Therapeutics, Merck, Sanofi, and Takeda Pharmaceuticals. SZU served in a consulting or advisory role for AbbVie, Amgen, Celgene, GlaxoSmithKline, Janssen, Karyopharm Therapeutics, Merck, Seattle Genetics, Skyline Diagnostics, and Takeda, served on speakers bureaus for Celgene, Janssen, Sanofi, and Takeda, and received research funding from Amgen, Array BioPharma, Bristol Myers Squibb, Celgene, GlaxoSmithKline, Janssen, Merck, Pharmacyclics, Sanofi, Seattle Genetics, and Skyline Diagnostics. JGB served in a consulting or advisory role for Bluebird Bio, Bristol Myers Squibb, Celgene, CRISPR Therapeutics, Janssen, Karyopharm Therapeutics, Kite/Gilead, Legend Biotech, Secura Bio, Servier, and Takeda, and received research funding from AbbVie, Acetylon Pharmaceuticals, Amgen, Bluebird Bio, Bristol Myers Squibb, Celgene, Celularity, Constellation Pharmaceuticals, CURIS, EMD Serono, Genentech/ Roche, Glenmark, Ichnos Sciences, Janssen, Kesios Therapeutics, Lilly, Novartis, Poseida, Sanofi, Takeda, Teva, and Vivolux. KY is a consultant physician and receives honoraria from Janssen, GSK, Amgen Inc., Takeda, and Sanofi and research funding from Janssen, Takeda, and Sanofi. AL, JMS, KQ, MV, AB, JD, SN and SV are employed by Janssen and have restricted stock units and/or stock options. AG and $\mathrm{TN}$ are employed by Legend Biotech, USA.

Ethics approval The CARTITUDE-1 trial protocol was reviewed and approved by an independent ethics committee/institutional review board at all participating sites. All patients participating in the trial provided written informed consent. Similarly, the protocols of the clinical trials of daratumumab (POLLUX [10], CASTOR [11], and EQUULEUS [12]) were approved by local or independent institutional review board or ethics committees at participating sites and all patients provided written informed consent. The current analyses were conducted in accordance with a protocol and statistical analysis plan developed prior to the start of data analysis.
Consent to participate Not applicable.

Consent for publication Not applicable.

Availability of data and material Requests for access to the CARTITUDE-1 trial study data may be submitted through the Yale Open Data Access (YODA) Project site at http://yoda.yale.edu. The data sharing policy of Janssen Pharmaceutical Companies is available at https:// www.janssen.com/clinical-trials/transparency.

Code availability Not applicable.

Authors' contributions All authors were responsible for the study conception and design. AL, KQ, and $\mathrm{SN}$ were responsible for acquisition and analysis. All authors were responsible for interpretation of the data and revising it critically.

Open Access This article is licensed under a Creative Commons Attribution-NonCommercial 4.0 International License, which permits any non-commercial use, sharing, adaptation, distribution and reproduction in any medium or format, as long as you give appropriate credit to the original author(s) and the source, provide a link to the Creative Commons licence, and indicate if changes were made. The images or other third party material in this article are included in the article's Creative Commons licence, unless indicated otherwise in a credit line to the material. If material is not included in the article's Creative Commons licence and your intended use is not permitted by statutory regulation or exceeds the permitted use, you will need to obtain permission directly from the copyright holder. To view a copy of this licence, visit http://creativecommons.org/licenses/by-nc/4.0/.

\section{References}

1. Rajkumar SV. Multiple myeloma: 2020 update on diagnosis, riskstratification and management. Am J Hematol. 2020;95(5):548 67. https://doi.org/10.1002/ajh.25791.

2. Dimopoulos MA, Richardson PG, Moreau P, Anderson KC. Current treatment landscape for relapsed and/or refractory multiple myeloma. Nat Rev Clin Oncol. 2015;12(1):42-54.

3. Weisel KC, Martin T, Yong K, Qi K, Londhe A, Kobos R, et al. Characteristics and treatment patterns of triple-class exposed patients with relapsed/refractory multiple myeloma who participated in clinical trials of daratumumab [O127]. In: The 47th annual meeting of the european society of blood and marrow transplantation: physicians - oral sessions (O010-O169). Bone Marrow Transplant. 2021;56:137-8.

4. Gandhi UH, Cornell RF, Lakshman A, Gahvari ZJ, McGehee E, Jagosky MH, et al. Outcomes of patients with multiple myeloma refractory to CD38-targeted monoclonal antibody therapy. Leukemia. 2019;33(9):2266-75. https://doi.org/10.1038/ s41375-019-0435-7.

5. Mehra M, Vogel M, Valluri S, Nair S, Schecter J, Slowik R, et al. Patient characteristics, treatment patterns and outcomes in patients with triple class refractory multiple myeloma [E-poster \#1032]. In: 25th European Hematology Association (EHA) annual congress (Virtual). 11-14 Jun 2020. https://library.ehaweb.org/eha/ 2020/eha25th/293522. Accessed 1 Sept 2021.

6. Berdeja JG, Madduri D, Usmani SZ, Jakubowiak A, Agha M, Cohen AD, et al. Ciltacabtagene autoleucel, a BCMA-directed CAR T-cell therapy in patients with relapsed/refractory multiple myeloma (CARTITUDE-1): a phase $1 \mathrm{~b} / 2$ open-label study. Lancet. 2021;398(10297):314-324. 
7. Usmani SZ, Berdeja JG, Madduri D, Jakubowiak AJ, Agha ME, Cohen AD, et al. Ciltacabtagene autoleucel, a B-cell maturation antigen (BCMA)-directed chimeric antigen receptor T-cell (CAR$\mathrm{T})$ therapy, in relapsed/refractory multiple myeloma (R/R MM): updated results from CARTITUDE-1 [oral presentation]. In: 2021 ASCO Annual Meeting I. J Clin Oncol. 2021;39(15_suppl):8005.

8. Legend Biotech. U.S. Food and Drug Administration grants BCMA CAR-T cilta-cel priority review for the treatment for relapsed/refractory multiple myeloma [press release]. https:// www.legendbiotech.com/pdf/LEGN_PR_05262021.pdf. Accessed 28 May 2021.

9. Janssen. Janssen announces CAR-T therapy ciltacabtagene autoleucel (cilta-cel) accepted for accelerated assessment in Europe for the treatment of patients with heavily pretreated multiple myeloma [press release]. https://johnsonandjohnson.gcs-web.com/staticfiles/315911e1-8c26-4933-9707-295fc794bbdb. Accessed 12 Apr 2021.

10. ClinicalTrials.gov. A study comparing daratumumab, lenalidomide, and dexamethasone with lenalidomide and dexamethasone in relapsed or refractory multiple myeloma. https://clinicaltrials. gov/ct2/show/NCT02076009. Accessed 13 Jul 2021.

11. ClinicalTrials.gov. Addition of daratumumab to combination of bortezomib and dexamethasone in participants with relapsed or refractory multiple myeloma. https://clinicaltrials.gov/ct2/show/ NCT02136134. Accessed 13 Jul 2021.

12. ClinicalTrials.gov. A study of JNJ-54767414 (HuMax CD38) (anti-CD38 monoclonal antibody) in combination with backbone treatments for the treatment of patients with multiple myeloma. https://clinicaltrials.gov/ct2/show/NCT01998971. Accessed $13 \mathrm{Jul}$ 2021.

13. Li F, Morgan KL, Zaslavsky AM. Balancing covariates via propensity score weighting. J Am Stat Assoc. 2018;113(521):390-400.

14. Dimopoulos MA, Oriol A, Nahi H, San-Miguel J, Bahlis NJ, Usmani SZ, et al. Daratumumab, lenalidomide, and dexamethasone for multiple myeloma. N Engl J Med. 2016;375(14):1319-31.

15. Palumbo A, Chanan-Khan A, Weisel K, Nooka AK, Masszi T, Beksac M, et al. Daratumumab, bortezomib, and dexamethasone for multiple myeloma. N Engl J Med. 2016;375(8):754-66.

16. Chari A, Suvannasankha A, Fay JW, Arnulf B, Kaufman JL, Ifthikharuddin JJ, et al. Daratumumab plus pomalidomide and dexamethasone in relapsed and/or refractory multiple myeloma. Blood. 2017;130(8):974-81.

17. Hernán MA, Robins JM. Using big data to emulate a target trial when a randomized trial is not available. Am J Epidemiol. 2016;183(8):758-64.

18. Backenroth D. How to choose a time zero for patients in external control arms. Pharm Stat. 2021;20(4):783-92.

19. Berger VW, Sankoh A. Prognostic variables in clinical trials. Wiley StatsRef Stat Ref Online. 2014. https://doi.org/10.1002/ 9781118445112.stat07072.

20. Austin PC. Balance diagnostics for comparing the distribution of baseline covariates between treatment groups in propensity-score matched samples. Stat Med. 2009;28(25):3083-107.

21. Kumar S, Paiva B, Anderson KC, Durie B, Landgren O, Moreau $\mathrm{P}$, et al. International Myeloma Working Group consensus criteria for response and minimal residual disease assessment in multiple myeloma. Lancet Oncol. 2016;17(8):e328-46.

22. Phillippo DM, Ades AE, Dias S, Palmer S, Abrams KR, Welton NJ. Methods for population-adjusted indirect comparisons in health technology appraisal. Med Decis Mak. 2018;38(2):200-11.
23. Phillippo DM, Ades AE, Dias S, Palmer S, Abrams KR, Welton NJ. NICE DSU Technical Support Document 18: Methods for population-adjusted indirect comparisons in submission to NICE. http://nicedsu.org.uk/wp-content/uploads/2017/05/Popul ation-adjustment-TSD-FINAL.pdf. Accessed 29 Oct 2021.

24. Lee J, Chia K. Estimation of prevalence rate ratios for cross sectional data: an example in occupational epidemiology. BR J Ind Med. 1993;50(9):861.

25. Funk MJ, Westreich D, Wiesen C, Stürmer T, Brookhart MA, Davidian M. Doubly robust estimation of causal effects. Am J Epidemiol. 2011;173(7):761-7.

26. Kumar S, Durie B, Nahi H, Vij R, Dimopoulos MA, Kastritis E, et al. Propensity score matching analysis to evaluate the comparative effectiveness of daratumumab versus real-world standard of care therapies for patients with heavily pretreated and refractory multiple myeloma. Leuk Lymphoma. 2019;60(1):163-71.

27. Jabbour EJ, Sasaki K, Ravandi F, Short NJ, Garcia-Manero G, Daver $\mathrm{N}$, et al. Inotuzumab ozogamicin in combination with low-intensity chemotherapy (mini-HCVD) with or without blinatumomab versus standard intensive chemotherapy (HCVAD) as frontline therapy for older patients with Philadelphia chromosome-negative acute lymphoblastic leukemia: a propensity score analysis. Cancer. 2019;125(15):2579-86.

28. Chin CK, Lim K, Lewis K, Jain P, Qing Y, Feng L, et al. Autologous stem cell transplantation for untreated transformed indolent B-cell lymphoma in first remission: an international, multi-centre propensity-score-matched study. Br J Haematol. 2020;191(5):806-15.

29. Lonial S, Lee HC, Badros A, Trudel S, Nooka AK, Chari A, et al. Belantamab mafodotin for relapsed or refractory multiple myeloma (DREAMM-2): a two-arm, randomised, open-label, phase 2 study. Lancet Oncol. 2020;21(2):207-21.

30. Lonial S, Lee HC, Badros A, Trudel S, Nooka AK, Chari A et al. Longer term outcomes with single-agent belantamab mafodotin in patients with relapsed or refractory multiple myeloma: 13-month follow-up from the pivotal DREAMM-2 study. Cancer. 2021;127(22):4198-12.

31. Chari A, Vogl DT, Gavriatopoulou M, Nooka AK, Yee AJ, Huff $\mathrm{CA}$, et al. Oral selinexor-dexamethasone for triple-class refractory multiple myeloma. N Engl J Med. 2019;381(8):727-38.

32. Munshi NC, Anderson LD Jr, Shah N, Madduri D, Berdeja J, Lonial S, et al. Idecabtagene vicleucel in relapsed and refractory multiple myeloma. N Engl J Med. 2021;384(8):705-16.

33. Ma X, Long L, Moon S, Adamson BJ, Baxi SS. Comparison of population characteristics in real-world clinical oncology databases in the US: Flatiron Health, SEER, and NPCR. medRxiv. 2020. https://doi.org/10.1101/2020.03.16.20037143.

34. Mateos M-V, Weisel K, Stefano VD, Perrot A, Donk NWCJvd, Goldschmidt $\mathrm{H}$, et al. A prospective, non-interventional, multinational study of real-life standard of care in patients with relapsed/ refractory multiple myeloma with $\geq 3$ prior lines of therapy: interim data from LocoMMotion [E-poster \#987]. In: European Hematology Association (EHA) Annual Congress (Virtual). 2021 Jun 9-17. https://library.ehaweb.org/eha/2021/eha2021-virtualcongress/325745. Accessed 1 Sept 2021.

35. Caers J, Paiva B, Zamagni E, Leleu X, Bladé J, Kristinsson S, et al. Diagnosis, treatment, and response assessment in solitary plasmacytoma: updated recommendations from a European Expert Panel. J Hematol Oncol. 2018;11(1):10. 


\section{Authors and Affiliations}

Katja Weisel ${ }^{1} \cdot$ Thomas Martin $^{2} \cdot$ Amrita Krishnan $^{3} \cdot$ Sundar Jagannath $^{4} \cdot$ Anil Londhe $^{5} \cdot$ Sandhya Nair $^{6}$. Joris Diels ${ }^{7}$. Martin Vogel $^{8} \cdot$ Jordan M. Schecter ${ }^{9} \cdot$ Arnob Banerjee $^{10}$. Jesus G. Berdeja ${ }^{11}$. Tonia Nesheiwat $^{12}$. Ashraf Garrett ${ }^{12}$. Keqin $\mathrm{Qi}^{13}$. Satish Valluri ${ }^{14}$. Saad Z. Usmani ${ }^{15} \cdot$ Kwee Yong $^{16}$

1 Section of Pneumology, Department of Oncology, Hematology and Bone Marrow Transplantation, University Medical Center Hamburg-Eppendorf, Martinstrasse 52, 20246 Hamburg, Germany

2 Department of Medicine, UCSF Helen Diller Family Comprehensive Cancer Center, San Francisco, CA, USA

3 Hematology/Hematopoietic Cell Transplantation, Judy and Bernard Briskin Center for Multiple Myeloma Research, Duarte, CA, USA

4 Department of Medicine, Mount Sinai Medical Center, New York, NY, USA

5 Clinical Biostatistics, Janssen R\&D, LLC, Titusville, NJ, USA

6 Health Economics Design \& Analytics, Janssen Pharmaceutica NV, Beerse, Belgium

7 Market Access Analytics and Health Economics, Janssen Pharmaceutica NV, Beerse, Belgium

8 Oncology EMEA, Janssen Global Services, LLC, Raritan, NJ, USA
9 Clinical Development, Cellular Therapy Program, Janssen R\&D, Raritan, NJ, USA

10 Clinical Research, Oncology Early Development, Janssen R\&D, Raritan, NJ, USA

11 Center for Blood Cancers, Sarah Cannon Research Institute, Nashville, TN, USA

12 Medical Affairs, Cell Therapy, Legend Biotech USA, Inc, Piscataway, NJ, USA

13 Real World Evidence, Janssen R\&D, LLC, Titusville, NJ, USA

14 Market Access, Janssen Global Services, LLC, Raritan, NJ, USA

15 Department of Hematologic Oncology and Blood Disorders, Levine Cancer Institute-Atrium Health, Charlotte, NC, USA

16 Hematology Department, University College Hospital, London, UK 